\title{
QR-RLS algorithm for error diffusion of color images
}

\author{
Gozde Bozkurt Unal \\ North Carolina State University \\ Electrical and Computer Engineering \\ Department \\ Raleigh, North Carolina 27695 \\ Yasemin Yardimci \\ Middle East Technical University \\ Informatics Institute \\ Ankara 06531, Turkey \\ Orhan Arikan \\ A. Enis Çetin \\ Bilkent University \\ Electrical Engineering Department \\ Ankara 06533, Turkey
}

\begin{abstract}
Printing color images on color printers and displaying them on computer monitors requires a significant reduction of physically distinct colors, which causes degradation in image quality. An efficient method to improve the display quality of a quantized image is error diffusion, which works by distributing the previous quantization errors to neighboring pixels, exploiting the eye's averaging of colors in the neighborhood of the point of interest. This creates the illusion of more colors. A new error diffusion method is presented in which the adaptive recursive leastsquares (RLS) algorithm is used. This algorithm provides local optimization of the error diffusion filter along with smoothing of the filter coefficients in a neighborhood. To improve the performance, a diagonal scan is used in processing the image. () 2000 Society of Photo-Optical Instrumentation Engineers. [S0091-3286(00)00611-5]
\end{abstract}

Subject terms: halftones; color dithering; displays; printing; quantization; QR-RLS algorithm.

Paper 990163 received Apr. 14, 1999; revised manuscript received June 2, 2000; accepted for publication June 7, 2000.

\section{Introduction}

Color output devices such as halftone color printers and palette-based displays are capable of producing only a limited number of colors, whereas the human eye can distinguish around 10 million colors under optimal viewing conditions. ${ }^{1}$ The eye perceives only a local spatial average of the color spots produced by a printing device and is relatively insensitive to errors made in high frequencies in an image. ${ }^{1}$ Halftoning algorithms therefore aim to preserve these local averages while forcing the errors between the continuous tone image and the halftone image to highfrequency regions. Existing halftoning techniques can be broadly classified as ordered dither, error diffusion, and optimization-based halftoning techniques. A comparative study of earlier image reproduction techniques can be found in Stoffel and Moreland. ${ }^{2}$

Ordered dither techniques are mainly based on thresholding each pixel value after adding a pseudonoise sequence. These techniques are attractive in the sense that they are simple to implement and computationally inexpensive because they require pixelwise operations. However, ordered dithering results in regular and periodic error patterns, which lowers the quality of the output image.

Another group of halftoning methods are error diffusion techniques first introduced by Floyd and Steinberg. ${ }^{3}$ They proposed an algorithm that is predicated on distributing the quantization error of the current pixel to neighboring pixels. Typically, at each pixel, the weighted sum of previous quantization errors is added to the current pixel value, and then the pixel is quantized to produce the output pixel value. These weights form an error diffusion filter. Error diffusion aims to preserve the local average value of the image, therefore a unity gain low-pass finite impulse response (FIR) filter is used for distributing the error.
Error diffusion was first developed for gray-scale images. For color images, error diffusion can be applied to each color component independently or a color pixel can be error diffused in a vectorized manner.

Some directional artifacts seen in error diffusion are due largely to the traditional raster of processing. ${ }^{4}$ Previous approaches for improving error diffusion employed various choices of space filling curves to define the order of processing, such as serpentine curves, ${ }^{4}$ Peano curves, ${ }^{5}$ and random space-filling curves. ${ }^{6}$

In contrast to deterministic error filter kernels, some recent research employed dynamically adjusting the error filter kernel using adaptive signal processing techniques. Akarun et al. ${ }^{7}$ used a vectorized error diffusion approach, and updated the error diffusion filter coefficients adaptively. Wong ${ }^{8}$ minimizes a local frequency-weighted error criterion to adjust the error diffusion kernel dynamically using the well-known least mean-squares (LMS) algorithm. ${ }^{9}$ Kollias and Anastassiou ${ }^{10}$ used neural networks to minimize a frequency-weighted mean-squared-error criterion.

In optimization-based halftoning techniques, the problem of halftoning is formulated as an optimization problem that minimizes an error metric between the continuous tone original image and its halftone version. Disadvantages of optimization-based methods for halftoning are that there are multiple optima, the methods are iterative, and they require substantially high computational power. For color images, processing requirements further increase.

Some hybrid schemes that combine different aspects of halftoning methods are proposed in the literature such as the blue-noise halftoning, ${ }^{11}$ green-noise halftoning, ${ }^{12}$ randomized error propagation, ${ }^{13}$ and using a nonlinear Laplacian operator. ${ }^{14}$ 


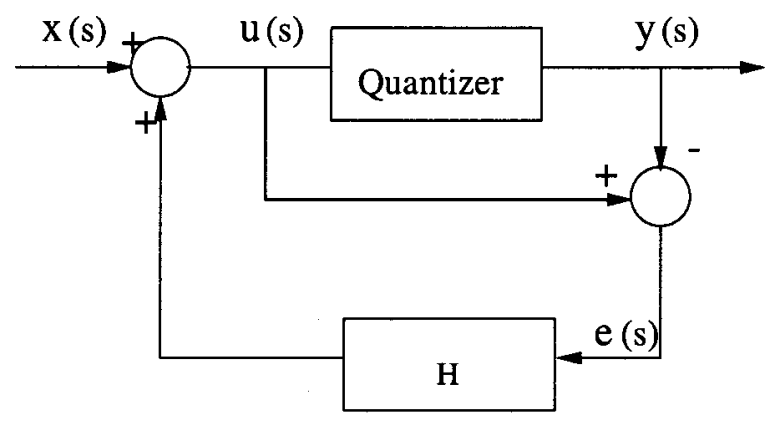

Fig. 1 Block diagram of the error diffusion method.

\section{Diagonal Error Diffusion}

A block diagram of the standard error diffusion technique is given in Fig. 1. Usually, the image is processed in a raster scan fashion, and each input color pixel $\mathbf{x}(s)$ is a 3 $\times 1$ vector, where the index $s=s_{1} M+s_{2}$ and $M$ is the number of horizontal pixels in the image. The current pixel $\mathbf{x}(s)$ together with the diffused error is quantized. The resultant image $\mathbf{y}(s)$ is the dithered image.

Here, $\mathbf{Q}$ is the quantizer, and $\mathbf{H}$ is the error diffusion matrix. Some well-known error diffusion filter masks ${ }^{3,15}$ are shown in Fig. 2, where the solid dots denote the pixel located at $s$. These masks determine the support of the error diffusion filter. A common characteristic of these filters is that they are casual, i.e., their region of support is wedge to ensure that these filters can be applied in a sequential manner. ${ }^{16}$ The filter coefficients are deterministic, low pass in nature, and add up to 1 so that errors are neither amplified nor reduced.

The pixel at location $s$ has the value

$\mathbf{u}(s)=\mathbf{x}(s)+\mathbf{H}(s) \mathbf{e}_{p}(s)$,

before quantization. Here, the error diffusion filter $\mathbf{H}$ has size $K \times L$, where $K$ is the number of channels, $N$ is the size of support of the error diffusion filter on each channel, and $L=K N$. For an RGB image that uses an error diffusion filter support of size 4 as the Floyd-Steinberg filter, $K=3$ and $N=4$. The composite error vector $\mathbf{e}_{p}(s)$ consists of the past quantization errors that must be diffused on the pixel at location $s$

$\mathbf{e}_{p}(s)=\left[\mathbf{e}_{1 p}^{T}(s) \mathbf{e}_{2 p}^{T}(s) \ldots \mathbf{e}_{K p}^{T}(s)\right]^{T}$,

where the subscript $p$ indicates that the quantization errors are made in the past. The past quantization error vector for the $k^{\prime}$ th channel $\mathbf{e}_{k p}(s)$ has the open form:

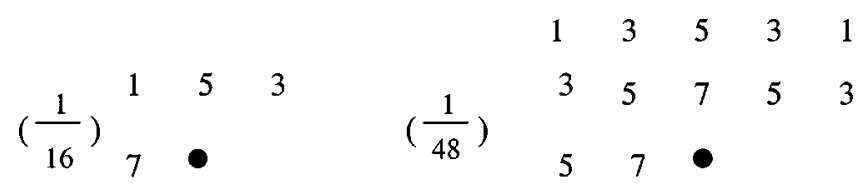

Floyd - Steinberg

Jarvis, Judice and Ninke

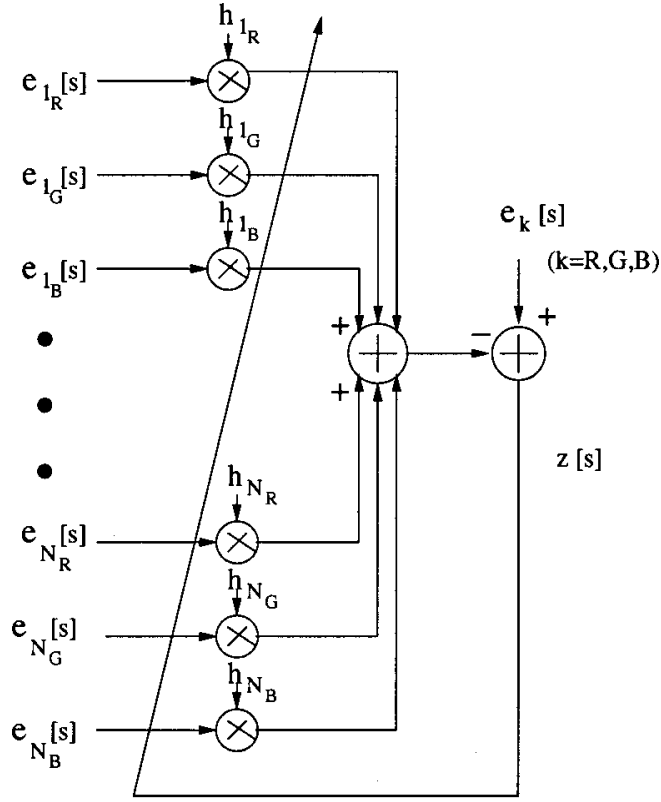

Fig. 3 One channel of the composite-multichannel error diffusion; where $N$ is the error diffusion filter size and $k$ is the channel index, which takes a value from the set $R=1, G=2$, and $B=3$.

$\mathbf{e}_{k p}(s)=\left[e_{1 k}(s) e_{2 k}(s) \ldots e_{N k}(s)\right]^{T}$,

where $e_{n k}(s)$ is the quantization error in the $k^{\prime}$ th channel for the $n$ 'th neighbor of the pixel $s$. There are two cases of interest:

1. Matrix $\mathbf{H}$ is a full matrix so that errors in different channels may be diffused on each other. We refer to this case as composite-multichannel error diffusion and its block diagram is depicted in Fig. 3. It may be also called vectorized error diffusion. ${ }^{7}$

2. Matrix $\mathbf{H}$ has the following block diagonal structure:

$$
\mathbf{H}=\left[\begin{array}{ccccc}
\mathbf{h}_{11}^{T} & 0 & 0 & \ldots & 0 \\
0 & \mathbf{h}_{22}^{T} & 0 & \ldots & 0 \\
\vdots & \vdots & \vdots & \ddots & 0 \\
0 & 0 & 0 & \ldots & \mathbf{h}_{K K}^{T}
\end{array}\right],
$$

with each $\mathbf{h}_{k k}^{T}$ designating the error diffusion filter for the $k$ 'th channel as follows:

$$
\mathbf{h}_{k k}^{T}=\left[h_{k 1} h_{k 2} \ldots h_{k N}\right] \text {. }
$$

In this structure, the errors in one channel are only diffused in the same channel. We call this strategy channel-bychannel error diffusion and its block diagram is given in Fig. 4. It is also called scalar error diffusion.

After error is diffused on the pixel $s,(K \times 1)$ quantization error vector $\mathbf{e}(s)$ for this pixel is formed as

$\mathbf{e}(s)=\mathbf{u}(s)-\mathbf{y}(s)$,

$\mathbf{y}(s)=Q[\mathbf{u}(s)]$.

Fig. 2 Error diffusion filter masks. 


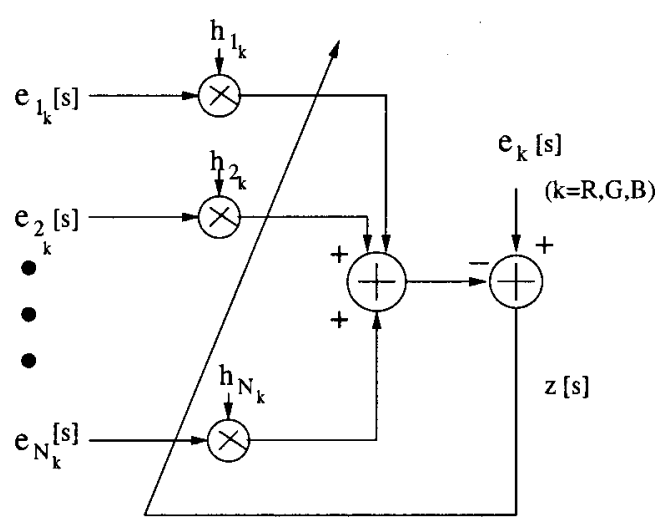

Fig. 4 One channel of the channel-by-channel error diffusion: $N$ is the error diffusion filter size and $k$ is the channel index which takes a value from the set $R=1, G=2$, and $B=3$.

The error between the original input pixel and the output pixel is defined as the output error,

$\mathbf{z}(s)=\mathbf{x}(s)-\mathbf{y}(s)$,

which can be expressed in terms of the past and the present quantization errors as

$\mathbf{z}(s)=\mathbf{e}(s)-\mathbf{H}(s) \mathbf{e}_{p}(s)$,

by substituting Eqs. (1) and (5) in Eq. (7).

The normal raster used in error diffusion causes vertical or horizontal artifacts, and regular patterns that arise especially in uniform intensity regions. It is well known that the human visual system is less sensitive to diagonal errors compared to the vertical or horizontal errors. To take advantage of this fact we scanned the image diagonally, hence the error is diagonally diffused. Causal prediction windows shown in Fig. 5 are used in the error diffusion algorithm. Here, we aim to break up the horizontal and vertical directionality of the possible error patterns, and force the accumulation of the error to be in diagonal orientation to which the human eye is less sensitive.

\section{New Adaptive Error Diffusion}

The error diffusion filter plays an important role in shaping the output error spectrum. In contrast to deterministic error diffusion filters, recent algorithms use the optimum filter

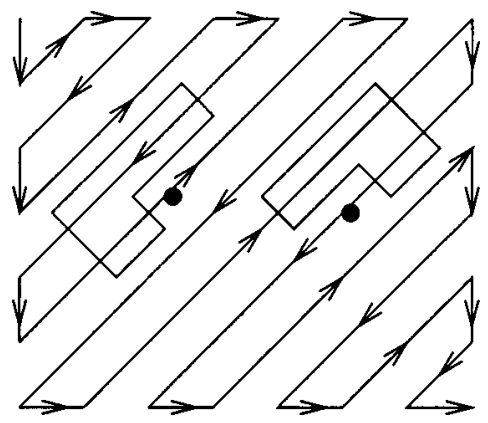

Fig. 5 Diagonal scanning: dots correspond to the current pixel, and the L-shaped window contains the previous pixels. coefficients for a given image, or update the coefficients adaptively using LMS type adaptive algorithms. ${ }^{7,8}$

We would like to minimize the energy of the output error $\mathbf{z}(s)$

$$
E\left[\|\mathbf{z}(s)\|^{2}\right]=E\left[\|\mathbf{x}(s)-\mathbf{y}(s)\|^{2}\right]=E\left[\left\|\mathbf{e}(s)-\mathbf{H}(s) \mathbf{e}_{p}(s)\right\|^{2}\right],
$$

with respect to the filter coefficients $\mathbf{H}(s)$. Since typical image characteristics are locally nonstationary, an adaptive algorithm is used for the minimization of the output error sequence energy. However, to reduce the effects of noise and provide further averaging of the filter coefficients around $s$ we included the past quantization errors around $s$ in our cost function. We propose to use a recursive leastsquares (RLS) type adaptation algorithm minimizing a cost function of the form

$J_{s}(\mathbf{H})=\sum_{j=1}^{s} \lambda^{s-j}\|\mathbf{z}(j)\|^{2}$,

where $\lambda \in[0,1]$ is the forgetting factor. The main advantages of this algorithm are the following:

1. fast adaptation to local features with an appropriately selected forgetting factor $\lambda$

2. numerical stability due to its lack of sensitivity to the condition matrix of the quantization error autocorrelation matrix

3. one-step convergence to the optimum $\mathbf{H}(s)$ matrix

RLS algorithms that employ QR decomposition of the quantization error autocorrelation matrix, also called QRRLS algorithms, have the added benefit of having the ability to work in low-bit arithmetic, therefore making them feasible to be implemented using very large scale integration (VLSI).

\subsection{Composite Multichannel Error Diffusion}

Substituting the expression for the output error in Eq. (10) we obtain

$$
\begin{aligned}
J_{s}(\mathbf{H}) & =\sum_{j=1}^{s} \lambda^{s-j}\left\|\mathbf{e}(j)-\mathbf{H}(j) \mathbf{e}_{p}(j)\right\|^{2} \\
& =\sum_{k=1}^{K} \sum_{j=1}^{s} \lambda^{s-j}\left[e_{k}(j)-\mathbf{h}_{k}^{T}(j) \mathbf{e}_{p}(j)\right]^{2} \\
& =\sum_{k=1}^{K} J_{k, s}\left(\mathbf{h}_{k}\right)
\end{aligned}
$$

where the rows of $\mathbf{H}(s)$ are denoted by $\mathbf{h}_{k}^{T}$. The minimization of the cost function $\mathbf{J}_{s}(\mathbf{H})$ with respect to the error diffusion filter coefficients matrix $\mathbf{H}(s)$ can be carried out by determining the rows $\mathbf{h}_{k}^{T}$ of the optimum matrix $\mathbf{H}(s)$ by minimizing the individual cost functions $J_{k, s}\left(\mathbf{h}_{k}\right)$ with respect to $\mathbf{h}_{k}$, for $k=1,2, \ldots, K$. The optimum solution has the form 
$\mathbf{h}_{k}=\mathbf{C}^{-1}(s) \boldsymbol{\Delta}_{k}(s)$,

where $\mathbf{C}(s)$ and $\boldsymbol{\Delta}_{k}(s)$ are the correlation estimates defined as

$\mathbf{C}(s)=\sum_{j=1}^{s} \lambda^{s-j} \mathbf{e}_{p}(j) \mathbf{e}_{p}^{T}(j)$,

$\boldsymbol{\Delta}_{k}(s)=\sum_{j=1}^{s} \lambda^{s-j} \mathbf{e}_{p}(j) e_{k}(j)$,

and the latter correlation has to be computed for each channel $k=1,2, \ldots, K$.

In QR-RLS the optimal solution of Eq. (12) is computed by employing a recursive procedure operating on the square-rooted covariance matrices providing numerical robustness even when very few bits are used for arithmetic implementations. Introducing the unique upper triangular Cholesky factor with positive diagonal entries $\mathbf{R}(s)$, and vectors $\boldsymbol{\Gamma}_{k}(s)$ :

$\mathbf{C}(s)=\mathbf{R}^{T}(s) \mathbf{R}(s)$,

$\boldsymbol{\Gamma}_{k}(s)=\mathbf{R}^{-T}(s) \boldsymbol{\Delta}_{k}(s)$,

the optimal error diffusion filter coefficients can be obtained as a function of the square-rooted covariance

$\mathbf{h}_{k}^{\star}(s)=\mathbf{R}^{-1}(s) \boldsymbol{\Gamma}_{k}(s), \quad k=1,2, \ldots K$.

The QR-RLS algorithm is effective because it can be implemented in a recursive manner. Assuming the matrices $\mathbf{R}(s-1)$ and $\mathbf{R}^{T}(s-1)$ and the vectors $\boldsymbol{\Gamma}_{k}(s-1)$ are already computed for the previous pixel $(s-1)$, they can be updated so that

$$
\begin{aligned}
\mathbf{Q}(s) & {\left[\begin{array}{ccc}
\sqrt{\lambda} \mathbf{R}(s-1) & \sqrt{\lambda} \boldsymbol{\Gamma}_{k}(s-1) & \frac{\mathbf{R}^{-T}(s-1)}{\sqrt{\lambda}} \\
\mathbf{e}_{p}^{T}(s) & e_{k}(s) & \mathbf{0}^{T}
\end{array}\right] } \\
& =\left[\begin{array}{ccc}
\mathbf{R}(s) & \boldsymbol{\Gamma}_{k}(s) & \mathbf{R}^{-T}(s) \\
\mathbf{0}^{T}(s) & \tilde{f}_{k}(s) & \widetilde{\mathbf{g}}^{T}(s)
\end{array}\right],
\end{aligned}
$$

with the vector $\tilde{\mathbf{g}}(s)$ and the variable $\widetilde{f}_{k}(s)$ updating the present optimum error diffusion filter as

$\mathbf{h}_{k}^{\star}(s)=\mathbf{h}_{k}^{\star}(s-1)-\widetilde{\mathbf{g}}(s) \widetilde{f}_{k}(s) \quad k=1,2, \ldots K$.

The orthonormal matrix $\mathbf{Q}(s)$ is of size $(L+1) \times(L+1)$ and consists of $L$ Givens rotations

$\mathbf{Q}(s)=\mathbf{Q}_{L}(s) \mathbf{Q}_{L-1}(s) \ldots \mathbf{Q}_{1}(s)$,

each with the form

$$
\mathbf{Q}_{i}(s)=\left[\begin{array}{cccc}
\mathbf{I}_{i-1} & \vdots & & \vdots \\
\cdots & \cos \theta_{i}(s) & \cdots & \sin \theta_{i}(s) \\
& \vdots & \mathbf{I}_{L-i} & \vdots \\
\cdots & -\sin \theta_{i}(s) & \cdots & \cos \theta_{i}(s)
\end{array}\right] .
$$

The Givens rotation matrices $\mathbf{Q}_{i}(s)$ differ from an identity matrix of size $(L+1) \times(L+1)$ only at its four entries, as shown in Eq. (19). The rotation angle $\theta_{i}(s)$ is selected so that the $(L+1, i)$ element of the matrix to which $\mathbf{Q}_{i}(s)$ is applied is annihilated while $\mathbf{R}(s)$ remains upper triangular. The $\mathbf{Q}_{i}(s)$ matrix constructed in this manner simultaneously updates the matrix $\mathbf{R}^{T}(s-1)$ and the vector $\boldsymbol{\Gamma}_{k}(s)$ as indicated by the last two columns of Eq. (18). The iterations can be started with a diagonal matrix $\mathbf{R}(0)=\sqrt{\delta} \mathbf{I}_{m}$. Yang and Böhme have described this and other rotationbased RLS algorithms in a unified framework. ${ }^{17}$

\subsection{Channel-by-Channel Error Diffusion}

In the previous subsection, error diffusion by using previously made errors in all of the channels is discussed. By constraining the form of the error diffusion coefficient matrix $\mathbf{H}(s)$, as in Eq. (4), further savings in computational load can be achieved with tolerable degradation in the overall performance of the diffusion process. In this case, the cost function of Eq. (11) simplifies to

$$
\begin{aligned}
\mathbf{J}_{s}(\mathbf{H}) & =\sum_{j=1}^{s} \lambda^{s-j}\left\|\mathbf{e}(j)-\mathbf{H}(j) \mathbf{e}_{p}(j)\right\|^{2} \\
& =\sum_{k=1}^{K} \sum_{j=1}^{s} \lambda^{s-j}\left[e_{k}(j)-\mathbf{h}_{k k}^{T}(j) \mathbf{e}_{p k}(j)\right]^{2} \\
& =\sum_{k=1}^{K} J_{k, s}\left(\mathbf{h}_{k k}\right),
\end{aligned}
$$

where the previous quantization errors for the $k$ 'th channel are given by

$\mathbf{e}_{p k}(j)=\left[e_{1 k}(n) e_{2 k}(n) \ldots e_{N k}(n)\right]^{T} \quad k=1,2, \ldots, K$.

We deliberately skipped the subscript $p$ on the right-hand side to simplify the notation.

\subsection{Computational Complexity}

The computational complexity of one iteration step of the standard LMS algorithm is known to be the length of the input vector for every channel. Therefore, the computational load of composite multichannel error diffusion is $K^{2} N$ and the load reduces to $L=K N$ for the channel-bychannel error diffusion. Computational complexity of the QR-RLS algorithm is $\mathbf{O}\left(K^{2} N^{2}\right)$ for the composite multichannel error diffusion and it is $\mathbf{O}\left(K N^{2}\right)$ for the channelby-channel implementation. With typical selections of $K$ $=3$ and $N=4$, the load QR-RLS algorithm is not very high compared to the LMS algorithm. Furthermore, systolic ar- 

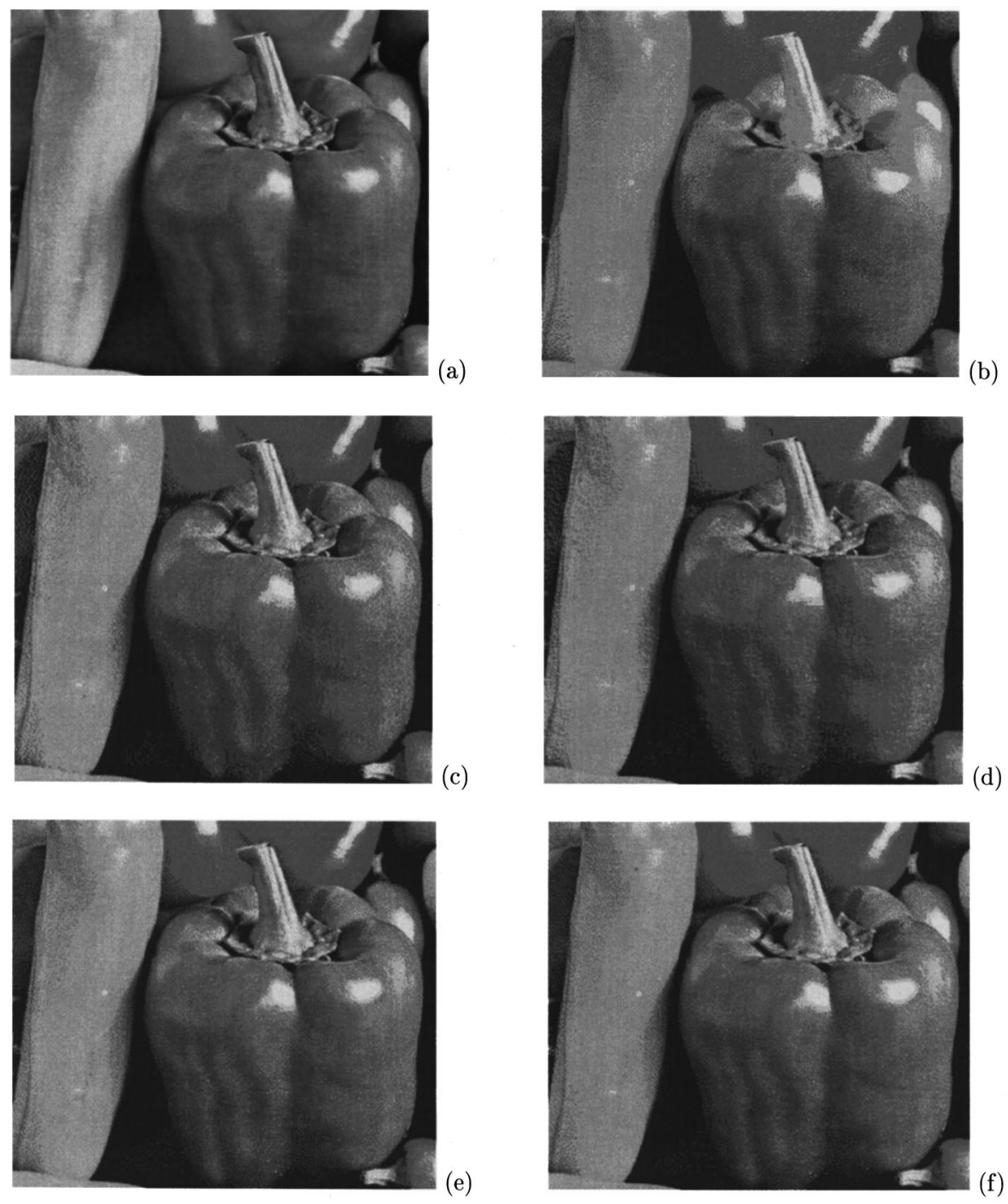

Fig. 6 (a) Original image, (b) standard error diffusion, (c) error diffusion with LMS (raster scan), (d) error diffusion with LMS (diagonal scan), (e) error diffusion with QR-RLS (raster scan), and (f) error diffusion with QR-RLS (diagonal scan).

rays can be utilized in the QR-RLS implementation and the proposed approaches can be realized efficiently by VLSI technology.

The diagonal and the traditional raster have the same computational load but the memory requirements may vary depending on the application. If the image is "scanned" diagonally the memory requirements only increase by a factor of $\sqrt{(2)}$, but when the image is already scanned horizontally almost the whole image has to be buffered.

\section{Simulation Results}

To demonstrate the performance of our error diffusion algorithm, we carried out simulations with several images among which a representative one is presented here. ${ }^{18}$ First the images were quantized to 16 levels using the median cut algorithm. ${ }^{19}$ We compared the new method with FloydSteinberg's method, and the adaptive error diffusion with LMS algorithm both with raster scan and diagonal scan of the image. Various values for the forgetting factor $\lambda$ for the QR-RLS algorithm were tried in the interval $[0.9,0.99]$ and the algorithm showed robust performance with these selections. We kept the forgetting factor as 0.95 in our simulations for both types of scanning methods. The parameter used in the initialization of the QR-RLS algorithm, $\sqrt{\delta}$, was chosen as 0.1 . The coefficients of the adaptive error diffusion filter in both algorithms were scaled by 0.9 , i.e., we allowed diffusion of not all but a fraction of the error made in quantization to neighboring pixels, and this resulted in a slight improvement in terms of color impulses. ${ }^{7}$

The results for the "Peppers" image is shown* in Fig. 6 . The image error diffused by Floyd-Steinberg's method in Fig. 6(b) contains color impulses, and the edges are smeared to each other. These artifacts, color impulses and false edges, are reduced in Figs. 6(c) and 6(d), which were obtained by the LMS-based error diffusion method. The image in Fig. 6(d) was obtained by diagonal processing which shows some improvement when compared to Fig.

\footnotetext{
*The color image outputs can be viewed in Ref. 20 .
} 


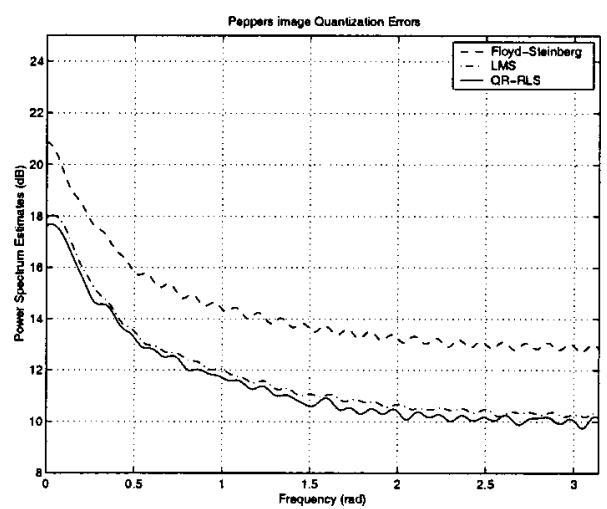

Fig. 7 Comparison of the quantization error spectra of one line.

6(c). However, in these images there are still false edges in slowly varying color regions. The images in Figs. 6(e) and 6(f) correspond to the vector adaptive error diffusion with QR-RLS algorithm. The resulting images obtained with the QR-RLS-based algorithm are sharper and brighter, giving the highest quality output. In Fig. 6(f), diagonal processing was used. We observe that this method shows the best performance since the color impulses and false contours are greatly eliminated.

It is pointed out in Ref. 21 that the whiter the quantization error power spectrum, the better the quality of the displayed image. We estimated the power spectrum of the quantization error for the resulting images by Welch's periodogram averaging method. ${ }^{22}$ The power spectrum of the quantization errors on a randomly selected horizontal line of the peppers image is shown in Fig. 7. In Figs. 8 and 9, the mean and the variance of the power spectrum of the quantization errors over all horizontal lines of the "Peppers" image are shown. As we can observe from these plots, the power spectrum of the quantization error diffused by the QR-RLS type adaptive method has not only the lowest energy but also the flattest response, whereas the error diffusion with Floyd-Steinberg has the highest energy.

We asked 14 independent viewers to give a subjective evaluation of three different images quantized to eight levels using the median cut algorithm when processed by dif-

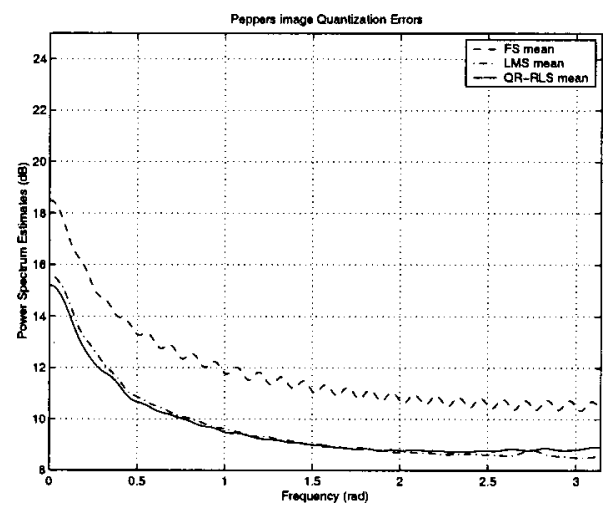

Fig. 8 Comparison of the mean quantization error spectra over all lines.

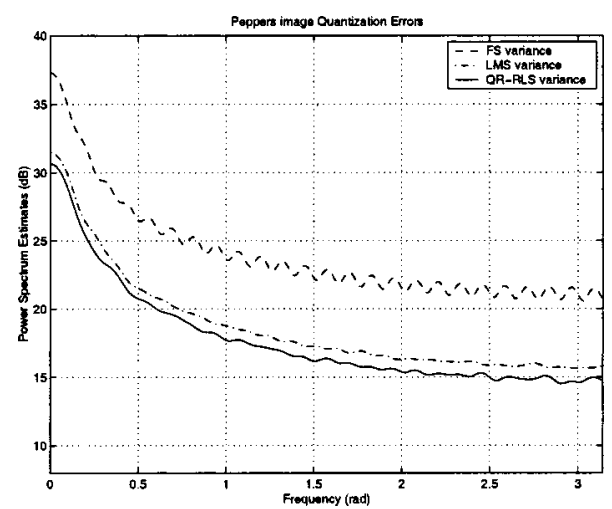

Fig. 9 Comparison of variance of the quantization error spectra over all lines.

ferent algorithms. All except one of our evaluators were graduate students in either electrical engineering or modeling and simulation. The last evaluator was a specialist in image processing. They were asked to grade the images from 10 (best) to 1 (worst) based on the similarity with the original as Mannos and Sakrison. ${ }^{23}$ The averages and the standard deviations of the grades are given in Table 1. We see that the LMS algorithm implementations are better than those of Floyd and Steinberg, but the QR-RLS-based methods are far superior. The diagonal implementation was slightly better than the nondiagonal for the LMS but the reverse was true for the QR-RLS. Even though the human visual system is less sensitive to diagonal artifacts, diffusing errors diagonally also implies diffusing errors onto pixels that are farther apart and therefore less correlated. This may offset the improvement the diagonal processing may provide.

\section{Conclusions}

We proposed a new adaptive error diffusion method for color images. We used the QR-RLS adaptive algorithm to update the error diffusion filter coefficients in the minimization of the weighted output error so that it is least noticeable to the human eye. We also exploited relative insensitivity of the human visual system to diagonal orientations, and scanned the image diagonally. Our simulation studies show that the new adaptive error diffusion algorithm outperforms the deterministic and LMS-type error diffusion algorithms.

Table 1 The results of subjective evaluation of three images.

\begin{tabular}{lccc}
\hline \hline \multicolumn{1}{c}{ Algorithm } & $\begin{array}{c}\text { Mean } \\
\text { Score }\end{array}$ & $\begin{array}{c}\text { Standard } \\
\text { Deviation }\end{array}$ & $\begin{array}{c}\text { Median } \\
\text { Score }\end{array}$ \\
\hline Floyd-Steinberg & 4.8095 & 1.2923 & 5 \\
LMS & 5.1905 & 1.3478 & 5 \\
LMS diagonal & 5.2143 & 1.2403 & 5 \\
QR-RLS & 7.2143 & 1.1590 & 7 \\
QR-RLS diagonal & 7.0714 & 1.3506 & 7 \\
\hline \hline
\end{tabular}


This paper was presented at the IEEE International Conf. on Image Processing (ICIP'98) and it was supported in part by the NATO Grant CRG-971117.

\section{References}

1. G. Sharma and H. J. Trussell, "Digital color imaging," IEEE Trans. Image Process. 6(7), 901-932 (1997).

2. J. C. Stoffel and J. F. Moreland, "A survey of electronic techniques for pictorial image reproduction," IEEE Trans. Commun. 29(12), 1898-1925 (1981)

3. R. W. Floyd and L. Steinberg, "An adaptive algorithm for spatial grayscale," 17, 75-77 (1976).

4. R. Ulichney, Digital Halftoning, MIT Press, MA (1987).

5. I. H. Witten and M. Neal, "Using peano curves for bilevel display of continuous tone images," IEEE Comput. Graph. Appl. 5, 47-52 (1982).

6. T. Asano, "Digital halftoning algorithm based on random spacefilling curve," in Proc. IEEE Int. Conf. on Image Processing, pp. 545-548 (1996).

7. L. Akarun, Y. Yardimci, and A. E. Cetin, "Adaptive methods for dithering color images," IEEE Trans. Image Process. 6(7), 950-955 (1997).

8. P. W. Wong, "Adaptive error diffusion and its application in multiresolution rendering," IEEE Trans. Image Process. 5(7), 1184-1196 (1996).

9. B. Widrow and S. D. Stearns, Adaptive Signal Processing, PrenticeHall, Englewood Cliffs, NJ (1985).

10. S. Kollias and D. Anastassiou, "A unified neural network approach to digital image halftoning," IEEE Trans. Signal Process. 39(4), 980894 (1991)

11. T. Mitsa and K. J. Parker, "Digital halftoning using a blue-noise mask," in Proc. IEEE Int. Conf. Acoust. Speech Signal Processing, pp. 2809-2802 (1991).

12. D. L. Lau, G. R. Arce, and N. C. Gallagher, "Green-noise digital halftoning," Proc. IEEE 86(12), 2424-2444 (1998).

13. G. Goertzel and G. R. Thompson, "Digital halftoning on the ibm 4250 printer," IBM J. Res. Dev. 31(1), $2-15$ (1987).

14. D. Anastassiou and K. S. Pennington, "Digital halftoning of images," IBM J. Res. Dev. 26(6), 687-697 (1982).

15. J. F. Jarvis, C. N. Judice, and W. H. Ninke, "A survey of techniques for the display of continous tone pictures on bilevel displays," Comput. Graph. Image Process. 5, 13-40 (1976).

16. J. S. Lim, Two-Dimensional Signal and Image Processing, PrenticeHall, Englewood Cliffs, NJ (1990).

17. B. Yang and J. F. Bohme, "Rotation-based RLS algorithms: unified derivations, numerical properties, and parallel implementations," IEEE Trans. Signal Process. 40(5), 1151-1167 (1992).

18. G. Bozkurt, "Novel methods in image halftoning,"' Master's Thesis, Bilkent University (1998).

19. P. Heckbert, "Color image quantization for frame buffer display," Comput. Graph. 16(7), 297-307 (1982)

20. http://www.ee.bilkent.edu.tr/ cetin/QRRLS

21. B. W. Kolpatzik and C. A. Bouman, "Optimized error diffusion for high-quality image display,"' J. Electron. Imaging 1(3), 277-292 (1992).

22. M. H. Hayes, Statistical Digital Signal Processing and Modeling, Wiley, New York (1996).
23. J. L. Mannos and D. J. Sakrison, "The effects of a visual fidelity criterion on the encoding of images," IEEE Trans. Inf. Theory 20(4), $525-536(1974)$

Gozde Bozkurt Unal received her BSc degree in electrical engineering from the Middle East Technical University, Ankara, Turkey, in 1996 and her MSc degree in electrical engineering from the Bilkent University, Ankara, Turkey, in 1998. She is currently a research assistant in the Electrical and Computer Engineering Department at North Carolina State University, Raleigh, pursuing her PhD degree.

Yasemin Yardimci received her BSc and MSc degrees from Bogazici University and her PhD degree from Vanderbilt University. She was an adjunct professor with Bilkent University and Istanbul University and a visiting assistant professor at the University of Minnesota in the 1996 to 1997 academic year. She is presently with the Faculty of Informatics, Institute of Middle East Technical University. She chairs the IEEE Aerospace and Electronic Systems Society chapter in Turkey.

Orhan Arikan received his BSc degree from Middle East Technical University, Ankara, Turkey, and his MSc and PhD degrees from the University of Illinois at Urbana-Champaign. He is currently with Bilkent University and chairs the IEEE Turkey section.

A. Enis Çetin studied electrical engineering at the Middle East Technical University. After receiving his BSc degree, he received his MSE and PhD degrees in systems engineering from the Moore School of Electrical Engineering at the University of Pennsylvania, Philadelphia. Between 1987 and 1989, he was an assistant professor of electrical engineering at the University of Toronto, Canada. Since then he has been with Bilkent University, Ankara, Turkey, where he is currently a full professor. During the summers of 1988 , 1991, and 1992 he was with Bell Communications Research, Bellcore, New Jersey. He spent the 1994 to 1995 academic year at Koc University in Istanbul, and the 1996 to 1997 academic year at the University of Minnesota, Minneapolis, as a visiting associate professor. Prof. Çetin is a member of the DSP technical committee of the IEEE Circuits and Systems Society and an associate editor of IEEE Transactions on Image Processing. He founded the Turkish chapter of the IEEE Signal Processing Society in 1991. He received the young scientist award of TUBITAK (Turkish Scientific and Technical Research Council) in 1993, chaired the IEEE-EURASIP Nonlinear Signal and Image Processing Workshop (NSIP'99) held in Antalya, Turkey, in June 1999, and organized the first IEEE Balkan Conference on Signal Processing, Communications and Circuits and Systems which was held in Istanbul in June 2000. 